\title{
Long-Term Survival in Patients with Inoperable and Metastasized Cancer
}

\author{
Siegfried Seeber \\ Department of Medical Oncology and Haematology, Kliniken Essen-Mitte, Huyssens- Stiftung, Essen, Germany
}

Patients with inoperable lung cancer, both of small cell and non-small cell types, have generally been associated with a fairly limited survival prognosis and, accordingly, current therapeutic standards concentrate on first- and second-line chemotherapies with or without radiotherapy.

In this issue of ONKOLOGIE, Gressner et al. [1] report on a patient with small cell lung cancer surviving 10 years after altogether 9 lines of chemotherapy. Primarily, a good partial remission had been achieved receiving 4 courses of carboplatinetoposide which had been afterwards consolidated, within a prospective study, by additional 4 courses of stem cell supported high-dose chemotherapy using the same substances. The induction therapy was finally completed by sequential radiation treatment.

In spite of this unusually aggressive induction therapy, only a 4-month progression-free interval was achieved, but the patient could successfully be kept alive at good quality of life with intermittent courses of topotecan, later gemcitabine, irinotecan, paclitaxel, docetaxel, thalidomide, additional afterloading radiation for bronchus obstruction, stent placement in the superior vena cava and finally by exposure to experimental radionuclide therapies.

This case report - although remarkable and a good lesson for less experienced oncologists whose therapeutic repertoire is often confined to established first- or second-line combinations (as they are recommended in the relevant guidelines by the various scientific societies in clinical oncology) - does not necessarily reflect a unique observation.

Many oncologists are nowadays used to bring to their patients everything that potentially helps, and the above-mentioned guidelines are no longer valid or helpful in cases where established therapy has failed. This holds also true for the scenario of registered versus non-registered ('off-label') therapy. Unfortunately, no guidelines on how to keep incurable patients alive as long and as good as possible have yet been defined al- though recommendations would be helpful for the treating physicians.

First-line therapy should, in most cases, be based on current study results and the best treatment should be first choice. But both the patient and his doctor should be aware that different strategies may sometimes offer similar, if not identical, results within the induction period in stage IV disease.

The effects of first-line therapy - as nicely demonstrated in the contribution by Gressner et al. - do not necessarily influence long-term prognosis even when using a very aggressive initial approach. Conversely, patients after less intensive induction might remain amenable to diverse strategies later. After consequent induction therapies the selection of later drugs and combinations appears to be restricted, but quite a number of options may be still available. At that stage, however, only in rare cases triple combinations would appear adequate, doublets are then most popular but the option of sequential mono-therapies (as shown in the case report) should also be considered. The repeated reinstitution of the same drug can indeed be a very good choice if the periods of disease stabilization are satisfactory and acute as well as cumulative toxicities are low. One of our patients with stage IV non-small cell lung cancer diagnosed in 11/1997 is still alive (S. Seeber and J. Hense, unpublished). Among other therapies, this patient has responded for about 6 years to gemcitabine, needing the drug as d1/d8/d15 q 4wks courses about 4-6 times a year in order to achieve the sequential partial remissions necessary for his continued symptom-free survival.

Under normal circumstances, after 18-24 months of chemotherapy at the latest, most approaches have to be regarded as experimental, not only because then treatment is considered to be off-label use. These patients continuously require close monitoring and early intervention at progression, a procedure which had been described as 'controlled off-label therapy'. Obviously, since such an approach should be based on rational

\begin{tabular}{|c|c|}
\hline KARGER & (C) 2008 S. Karger GmbH, Freiburg \\
\hline $\begin{array}{l}\text { Fax +49 } 7614520714 \\
\text { E-mail Information@Karger.de } \\
\text { www.karger.com }\end{array}$ & $\begin{array}{l}\text { Accessible online at: } \\
\text { www.karger.com/onk }\end{array}$ \\
\hline
\end{tabular}

Prof. Dr. Siegfried Seeber

Kliniken Essen Mitte, Evang. Huyssens Stiftung/Knappschaft GmbH

Klinik für Internistische Onkologie / Hämatologie

Henricistr. 92, 45136 Essen, Germany

Tel. +49 201 174-24701, Fax -24700

s.seeber@kliniken-essen-mitte.de 
grounds and on phase I-III data as well as personal experience a distinct expertise is mandatory in this area.

No patient can tolerate continued intensive therapies over years. Therefore, the importance of therapeutic breaks becomes obvious in such chronified courses. Opportunities for therapyfree intervals are given in periods of decreasing tumor parameters ('still responding'), at times of asymptomatic tumor stabilization ('continued partial remission') or, in selected situations such as hormone-refractory prostate cancer, also during closely observed phases of asymptomatic tumor marker progression ('low-dynamic, asymptomatic biochemical failure'). Quite similarly, in advanced ovarian cancer where survival now almost regularly reaches several years and protracted courses over 8-10 years are not uncommon, sometimes more than 20 therapeutic interventions have been applied at the time of CA125 progression [2]. In a variety of diseases such as PSA-positive prostate cancer, CA12-5-positive ovarian cancer or CA15-3positive breast cancer, the clinical course can be reliably followed by the tumor marker. However, it remains unclear whether interventions should be positioned at the very time of the tumor marker turnaround or, if asymptomatic, whether certain marker increments up to previous levels should be allowed. Such phases of 'controlled progression' possibly can positively affect overall survival, but most of these professional therapeutic maneuvers in long-term survivors, although helpful, cannot be based on current phase-III literature [2].

The question of maintenance therapies after complete or partial remissions has been a matter of controversy for a long time and contradictory study results exist. With he arrival of targeted therapies against epithelial and vascular tumor components evidence is increasing that remissions after single chemotherapeutic elements can be prolonged. In addition, an interposition of metronomic therapies between more aggressive phases of therapy is often helpful also for the patients' physical and psychical recovery.

As the case of Gressner et al. also beautifully demonstrates, long-term survivors not only need adequate drug therapies, such as dose-dense regimens, softer interims and breaks, but also adequate and personalized loco-regional interventions. The latter therapies are guided by symptoms and the prioritization of systemic versus local measures again requires high expertise in the multidisciplinary management.

In conclusion, survival of patients with common metastatic cancers is in most cases not determined by the choice of firstline chemotherapy but mainly by sequentially applied alternative systemic and local treatments. Since the landscape of clinical studies in oncology does not offer solutions for the numerous decisions necessary for individualized long-term patient management retrospective casuistic analyses are absolutely essential.

\section{References}

1 Gressner O, Popp H, Mey U, Friedrichs N, Strehl J, Sauerbruch T, Schmidt-Wolf IGH, Gorschlüter M: Long-term survival of a patient with small cell lung cancer after nine lines of chemotherapy and radiation. Onkologie 2008;31:469-472.

2 Seeber S, Braun AH: Phase III trials in oncology: setting standards of care? Nature Clinical Practice Oncology 2005;2:426-427. 